\title{
LYAPUNOV-TYPE INEQUALITY FOR QUASILINEAR SYSTEMS WITH ANTI-PERIODIC BOUNDARY CONDITIONS
}

\author{
Mustafa FAHri AKTAŞ, DEVRIM ÇAKMAK AND AYDin TIRYAKI
}

Abstract. In this paper, we establish a new Lyapunov-type inequality for quasilinear systems with the anti-periodic boundary conditions. It improves some result of Wang [17]. As an application, we also obtain lower bounds for the eigenvalues of corresponding systems.

Mathematics subject classification (2010): 34C10, 34B15, 34L15.

Keywords and phrases: Lyapunov-type inequality, anti-periodic boundary condition, lower bound.

\section{REFERENCES}

[1] G. A. Afrouzi, S. Heidarkhani, Existence of three solutions for a class of Dirichlet quasilinear elliptic systems involving the $\left(p_{1}, p_{2}, \ldots, p_{n}\right)$-Laplacian, Nonlinear Anal. 70 (2009), 135-143.

[2] M. F. Aktaş, D. ÇAKmaK, A. TiRyaki, A note on Tang and He's paper, Appl. Math. Comput. 218 (2012), 4867-4871.

[3] A. Canada, J. A. Montero, S. Villegas, Lyapunov inequalities for partial differential equations, J. Funct. Anal. 237 (2006), 176-193.

[4] A. CAnAdA, S. VIllegas, Optimal Lyapunov inequalities for boundary value problems, J. Math. Inequal. 3 (2009), 631-643.

[5] D. ÇАКМАК, Lyapunov-type integral inequalities for certain higher order differential equations, Appl. Math. Comput. 216 (2010), 368-373.

[6] D. ÇAKMAK, A. TIRYAKI, On Lyapunov-type inequality for quasilinear systems, Appl. Math. Comput. 216 (2010), 3584-3591.

[7] D. ÇAKMAK, A. TIRYAKI, Lyapunov-type inequality for a class of Dirichlet quasilinear systems involving the $\left(p_{1}, p_{2}, \ldots, p_{n}\right)$-Laplacian, J. Math. Anal. Appl. 369 (2010), 76-81.

[8] D. ÇAKMAK, On Lyapunov-type inequality for a class of nonlinear systems, Math. Inequal. Appl. 16 (2013), 101-108.

[9] L. Y. Chen, C. J. Zhao, W. S. Cheung, On Lyapunov-type inequalities for two-dimensional nonlinear partial systems, J. Inequal. Appl. 2010, Art. ID 504982, 12 pp.

[10] P. Hartman, Ordinary Differential Equations, Wiley, New York, 1964 an Birkhauser, Boston 1982.

[11] A. M. Liapunov, Probléme général de la stabilité du mouvement, Ann. Fac. Sci. Univ. Toulouse, 2 (1907), 203-407.

[12] P. L. NAPOli, J. P. Pinasco, Estimates for eigenvalues of quasilinear elliptic systems, J. Differential Equations 227 (2006), 102-115.

[13] X. H. TANG, X. HE, Lower bounds for generalized eigenvalues of the quasilinear systems, J. Math. Anal. Appl. 385 (2012), 72-85.

[14] A. TIRYAKI, D. ÇAKMAK, M. F. AKTAŞ, Lyapunov-type inequalities for a certain class of nonlinear systems, Comput. Math. Appl., 64 (2012), 1804-1811.

[15] X. YANG, Y. KIM, K. Lo, Lyapunov-type inequality for n-dimensional quasilinear systems, Math. Inequal. Appl. 16 (2013), 929-934.

[16] X. YAng, Y. Kim, K. Lo, Lyapunov-type inequality for quasilinear systems, Appl. Math. Comput. 219 (2012), 1670-1673.

[17] Y. WANG, Lyapunov-type inequalities for certain higher order differential equations with antiperiodic boundary conditions, Appl. Math. Letters 25 (2012), 2375-2380. 